\title{
Análisis Psicométrico de la Escala de Relaciones Intrafamiliares
}

\author{
Psychometric Analysis of the Intrafamily Relationships Scale
}

\author{
Remberto Castro Castañeda ${ }^{1}$, Esperanza Vargas Jiménez ${ }^{2}$, Silvana Mabel Núñez Fadda ${ }^{3}$, \\ Juan Evaristo Callejas Jerónimo ${ }^{4}$ y Gonzalo Musitu Ochoa ${ }^{5}$
}

\section{Resumen}

El objetivo de este artículo es presentar evidencias científicas de validez y fiabilidad de la Escala de Relaciones Intrafamiliares (E.R.I.). Participaron 1498 adolescentes escolarizados seleccionados a partir de un muestreo estratificado proporcional, de edades comprendidas entre los 10 y 15 años de edad $(M=12.49$. $D T=1.59$ ), de los cuales 728 fueron niños, y 770 niñas. Los resultados obtenidos a través del análisis exploratorio y confirmatorio muestran que la escala presenta una estructura clara y coherente de dos factores: unidad, apoyo y expresividad (factor 1) y dificultades familiares (factor 2). La fiabilidad es alta en la escala total y en ambos factores. Respecto de la validez, ambos factores tienen correlaciones significativas y coherentes con la literatura científica en las siguientes variables: actitudes hacia la autoridad institucional, transgresión de normas, conductas delictivas y violentas, violencia y victimización escolar. Se discuten los resultados obtenidos.

Palabras clave: Escala de Relaciones Intrafamiliares, actitudes hacia la autoridad, conductas delictivas, violencia y victimización escolar

\begin{abstract}
The aim of this article is to present scientific evidences of validity and reliability of the Intrafamily Relations Scale (I.R.S.). A total of 1498 school-aged adolescents participated, selected from a proportionate stratified sample, aged between 11 and 15 years, of whom 728 were boys and 770 girls. The results obtained through exploratory and confirmatory analysis show that the scale has a clear and coherent structure of two factors: unity, support and expressiveness (factor 1) and family difficulties (factor 2). Reliability is high on the full scale and on both factors. Regarding validity, both factors have significant and coherent correlations with the scientific literature in the following variables: attitudes towards institutional authority, transgression of norms, criminal and violent behavior, violence and school victimization. The results obtained are discussed.
\end{abstract}

Keywords: Intrafamily Relations Scale, attitudes towards authority, criminal behavior, violence and school victimization

\footnotetext{
${ }^{1}$ Doctor por la Universidad de Oviedo, España. Profesor Investigador Titular C del Departamento de Psicología del Centro Universitario de la Costa Campus Puerto Vallarta, de la Universidad de Guadalajara. Avenida Universidad de Guadalajara \#203, delegación Ixtapa Puerto Vallarta, Jalisco, México. C.P 48280. Tel.: 55 3222262455. Correo: reembert@ hotmail.com

2 Doctora Ciencias para el Desarrollo y Turismo por la Universidad de Guadalajara. Profesor Investigador Titular A del Departamento de Psicología del Centro Universitario de la Costa Campus Puerto Vallarta, de la Universidad de Guadalajara. Avenida Universidad de Guadalajara \#203, delegación Ixtapa Puerto Vallarta, Jalisco, México. C.P 48280. Tel.: 55 3222262455. Correo: esperanzavgas@hotmail.com

${ }^{3}$ Doctora en Ciencias para el Desarrollo, la Sustentabilidad y el Turismo por la Universidad de Guadalajara. Profesor Docente Asociado B del Departamento de Psicología del Centro Universitario de la Costa Campus Puerto Vallarta, de la Universidad de Guadalajara. Avenida Universidad de Guadalajara \#203, delegación Ixtapa Puerto Vallarta, Jalisco, México. C.P 48280. Tel.: 55 3222262455. Correo: drasilvananunez@gmail.com

${ }^{4}$ Doctor en Ciencias Sociales por la Universidad Pablo de Olavide. Investigador del Departamento de Educación y Psicología Social de la Universidad Pablo de Olavide, Sevilla, España. Carretera Utrera Km. 1. 41013. Sevilla, España. Correo: jecaljer@upo.es

${ }^{5}$ Doctor en Psicología por la Universidad de Valencia, España. Catedrático de Psicología Social de la Universidad Pablo de Olavide, Sevilla, España. Carretera Utrera Km. 1. 41013. Sevilla, España. Tel.: 34 963864420; Ext.: 6211. Correo: gmusoch@upo.es

Revista Iberoamericana de Diagnóstico y Evaluación - e Avaliação Psicológica. RIDEP · N58 · Vol.1 · 19-33 · 2021

ISSN: 1135-3848 print /2183-6051online
} 


\section{Introducción}

El funcionamiento familiar es una dimensión de gran trascendencia en la investigación social, clínica y neurobiológica por sus implicaciones en el desarrollo de niños, niñas y adolescentes. La influencia de los miembros de la familia, en especial de los padres, en la salud y el bienestar de sus hijos, a partir de uno de los procesos más importantes en las relaciones parentofiliales como es la socialización parental, se ha considerado como un hecho universal, que supera las diferencias culturales e históricas (Smetana, 2017; Stafford, Kuh, Gale, Mishra, \& Richards, 2016; Grusec, 2011).

El funcionamiento familiar, en sus diversas dimensiones, se ha relacionado con factores promotores y protectores en los ámbitos del desarrollo cognitivo y de modulación emocional (Whittle et al., 2016), así como de la salud mental y del bienestar positivo, desde la infancia hasta la senectud (Jin, Zhang, \& Han 2017; Stafford, et al., 2016).

En cuanto a la función socializadora, se han estudiado los vínculos de unas relaciones familiares adecuadas con el buen ajuste psicosocial de los hijos adolescentes, a través de indicadores individuales como la autoestima (García, Serra, Zacarés, \& García, 2018; Musitu \& Callejas, 2017; Rodríguez-Fernández, RamosDíaz, Ros, Fernández-Zabala, \& Revuelta, 2016), la satisfacción con la vida (Stafford, et al., 2016; Zhao, Xu, Wang, Jiang, \& Zhang, 2015), el ajuste escolar y el desempeño académico (Álvarez et al., 2015; Alves, Gomes, Martins, \& Almeida, 2017), la actitud positiva ante la autoridad y las normas sociales (Bonilla, Nuñez, Domínguez, \& Callejas, 2017; Carrascosa, Cava, \& Buelga, 2015; Jiménez, Estévez, \& Murgui, 2014), la empatía ambiental y la conexión con la naturaleza (Musitu-Ferrer, León-Moreno, Callejas-Jerónimo, Esteban-Ibáñez, \& Musitu-Ochoa, 2019).

Por el contrario, los problemas en el funcionamiento familiar se han relacionado con síntomas internalizantes y externalizantes en los hijos adolescentes (Wiggins, Mitchell, Hyde, \& Monk, 2015; García et al., 2018) como depresión (Thomassin, Suveg, Davis, Lavner, \& Beach, 2017; Freed, Rubenstein, Daryanani, Olino, \& Lauren, 2017), ansiedad (Simpson, Vannucci, \&
Ohannessian, 2018; Oppenheimer et al., 2016; León-Moreno, Callejas-Jerónimo, SuárezRelinque, Musitu-Ferrer, \& Musitu-Ochoa, 2020), violencia escolar (Garcés-Prettel, Santoya-Montes, \& Jiménez-Osorio, 2020; Romero-Abrio, MusituOchoa, Callejas-Jerónimo, Sánchez-Sosa, \& Villareal-González, 2018; Cerezo, Ruiz-Esteban, Lacasa, \& Arense, 2018; Bonilla, Nuñez, Domínguez, \& Callejas, 2017; Estévez, Jiménez, \& Cava, 2016), victimización escolar (Nuñez-Fadda, Castro-Castañeda, Vargas-Jiménez, Musitu-Ochoa, \& Callejas-Jerónimo, 2020; León-Moreno et al., 2020; Castro, Nuñez, Musitu, \& Callejas, 2019), uso de substancias (Riquelme, Garcia, \& Serra, 2018), ciberacoso (Romero-Abrio et al., 2019; Martínez-Ferrer et al., 2019) uso problemático del móvil (Santana-Vega, Gómez-Muñoz, \& FelicianoGarcía, 2019) conductas de riesgo sexual, así como conductas antisociales en los adolescentes (Johnson, Giordano, Longmore, \& Manning, 2016). Los argumentos expuestos respecto a la importancia de la familia en el bienestar del adolescente justifican la validación de este instrumento que evalúa con rigor -fiabilidad y validez- las dimensiones de funcionamiento familiar que impactan en su ajuste psicosocial (Hamilton \& Carr, 2016).

Gran parte de los instrumentos de medición más utilizados para evaluar el funcionamiento familiar se originaron en Estados Unidos, y parten de modelos teóricos particulares sobre el funcionamiento familiar, de manera que su estructura y factores están determinados por el modelo correspondiente. Para cada modelo, se diseñaron escalas de evaluación de autoinforme para la investigación del contexto clínico, tanto en versiones extensas como abreviadas. Las escalas más utilizadas actualmente en el ámbito anglosajón son: el Instrumento de evaluación familiar de McMaster (FAD: McMaster Family Assessment Device) de Epstein, Baldwin y Bishop (1983); la escala de FACES IV (Circumplex Model Family Adaptability and Cohesion Evaluation Scales, version IV); el SFI (Systems Model Self-Report Family Inventory) de Beavers y Hampson (2000) y la escala de Clima Familiar (FES: Family Environment Scale) de Moos y Moos (1981).

En el contexto latinoamericano, el desarrollo de escalas para evaluar el funcionamiento familiar 
ha sido muy limitado y de fecha reciente. La revisión de las publicaciones en español muestra que la escala sobre la que más se ha trabajado, en traducciones adaptadas, es la del modelo circunflejo de Olson (FACES), en sus versiones II, III y IV (Cracco \& Costa-Ball, 2019; MartínezPampliega, Merino, Iriarte, \& Olson, 2017; Schmidt, Barreyro, \& Maglio, 2010). En estos estudios se han señalado las diferencias de resultados en la estructura factorial, subrayando que las diferencias del contexto y del idioma de origen tienen un efecto importante respecto a la utilidad de estas escalas, diseñadas en otros ámbitos culturales. Un ejemplo es el trabajo del Grupo Lisis, investigadores que estudian la familia y el desarrollo adolescente en España, que han realizado adaptaciones y análisis psicométricos importantes en las escalas de evaluación del funcionamiento familiar, entre los que destacan la Escala de Comunicación PadresAdolescente (Barnes \& Olson, 1985), que evalúa la comunicación en tres dimensiones: Abierta, Ofensiva y Evitativa; la Escala de Clima Familiar (Moos \& Moos, 1981, adaptada por FernándezBallesteros \& Sierra, 1989) que mide Cohesión, Expresividad y Conflicto en las relaciones familiares; y el Cuestionario Family APGAR de Smilkstein, Ashworth y Montano (1982), adaptado por Bellón, Delgado, Luna y Lardelli (1996) que evalúa las características familiares de Adaptación, Participación, Gradiente de Crecimiento, Afecto y Resolución. Dichos instrumentos han sido validados también para el contexto Latinoamericano, y han contribuido enormemente en la comprensión de la importancia de las variables familiares en el buen ajuste de los hijos adolescentes.

Las familias mexicanas y de otros países de Latinoamérica han evolucionado a nuevas formas familiares, pero comparten el predominio de un modelo tradicional de funcionamiento, que asigna la mayor responsabilidad del cuidado y la crianza a las mujeres. Esto determina la mayor centralidad de la figura materna en el clima y la comunicación familiar, especialmente con niños y adolescentes (Comisión Económica para América Latina y el Caribe, 2016; Instituto de Política Familiar, 2019; Ullman, Maldonado Valera \& Rico, 2014).

En México, si bien han surgido diversas propuestas de escalas para evaluar el funcionamiento familiar (Calleja, 2011), son escasas las investigaciones respecto de las propiedades psicométricas de dichos instrumentos, así como del funcionamiento familiar y su relación con el ajuste psicosocial del adolescente. Ante esta carencia, se destaca la relevancia de proponer un instrumento de Relaciones Intrafamiliares con adecuadas propiedades psicométricas, diseñado específicamente para el contexto mexicano y latinoamericano, que ofrece la oportunidad y el estímulo para realizar investigaciones rigurosas de las relaciones familiares y el ajuste en el adolescente, enfatizando la importancia de los valores de los ámbitos locales y culturales (Smetana, 2017).

La Escala de Relaciones Intrafamiliares (E.R.I) fue elaborada en México por RiveraHeredia y Andrade (2010), con base en las escalas de Funcionamiento Familiar FES, la FACES II, la Escala de Atri y Zetune (1987), el FAD de MacMaster (Epstein, Baldwin, \& Bishop, 1983), la Escala de Hovestad sobre la Familia de Origen y la Escala de Funcionamiento Familiar de Bloom (Bloom, 1985). Para estos autores, el concepto de "relaciones intrafamiliares" puede equipararse al de ambiente o clima familiar y al de recursos familiares. Ambos se refieren a las conexiones recíprocas que se producen entre los miembros de cada familia, que comprenden las percepciones respecto a la unión familiar, el estilo de afrontamiento de problemas y de expresión de las emociones, el manejo de reglas y la adaptación a los cambios. En la escala original se obtuvieron tres dimensiones o factores: 1) Unión y Apoyo, que hace referencia a la disposición de la familia a convivir, realizar actividades compartidas y a proporcionarse apoyo mutuo; 2) Expresión, que mide la comunicación verbal de las emociones, ideas y acontecimientos de la familia en un ambiente de respeto; y 3) Dificultades, que agrupa los aspectos considerados problemáticos, negativos o difíciles, ya sea por el individuo o por la sociedad.

El primer estudio sobre las propiedades psicométricas de la E.R.I se publicó en 2010 (Rivera-Heredia \& Andrade 2010), pero no se han confirmado aún sus propiedades psicométricas y su validez. Estas carencias y la constatación de que la escala ofrecía datos de calidad (Bonilla, 
2016; Bonilla, Nuñez, Domínguez, \& Callejas, 2017) son las razones por las que se llevó a cabo el presente trabajo, con dos objetivos fundamentales: 1) Analizar la validez y fiabilidad de la escala E.R.I. y examinar su estructura factorial exploratoria y confirmatoria; 2) Estudiar las relaciones entre los distintos factores de la E.R.I y algunos indicadores de ajuste psicosocial en la adolescencia como las actitudes hacia la autoridad y las normas, la conducta violenta/disruptiva, y la violencia-victimización escolar.

\section{Método}

\section{Participantes}

En este estudio participaron un total de 1498 adolescentes mexicanos de ambos sexos (51.3\% hombres y $48.7 \%$ mujeres), con edades comprendidas entre los 10 y 15 años $(M=12.49$. $D T=1.59)$. La población de referencia $(N=19,457)$ fueron los estudiantes de $5 .^{\circ}$ y $6 .^{\circ}$ de primaria $(n=7,617)$ y los tres grados de secundaria de las escuelas públicas de Puerto Vallarta $(n=11,838)$. El tamaño de la muestra, para un intervalo de confianza del $3.5 \%$, con un nivel de confianza del 99\% y una varianza poblacional de .5, se fijó en 1,250. La selección de los participantes se realizó mediante un muestreo estratificado por afijación proporcional (Santos, Muñoz, Juez, \& Cortiñas, 2003); los estratos se determinaron en función del grado, a partir del listado de escuelas proporcionado por la autoridad educativa. Se determinaron los grupos proporcionales por escuela y grado de 98 escuelas, 72 primarias y 26 secundarias. Se excluyeron cuatro escuelas primarias con menos de 100 alumnos, con representación proporcional menor a un estudiante. Los participantes se seleccionaron de las listas de asistencia con una tabla de números al azar. Se incluyeron estudiantes de quinto $(n=291)$ y sexto grado de primaria $(n=298)$ y de primero $(n=325)$, segundo $(n=308)$ y tercer grado de secundaria $(n=284)$. Se excluyeron nueve sujetos porque no respondieron a más del $35 \%$ de los cuestionarios.

\section{Procedimiento}

Las escuelas fueron informadas sobre los objetivos de la investigación y se solicitó su colaboración, informando también a los padres de los alumnos sobre el estudio a través de un consentimiento por escrito. A los adolescentes se les indicó que su participación sería voluntaria y que las respuestas a los instrumentos serían anónimas. Durante la administración de los cuestionarios, además de los estudiantes entrenados para colaborar en esa tarea, un investigador del equipo de trabajo estuvo presente en el centro educativo.

El estudio cumplió los valores éticos requeridos en la investigación con seres humanos, respetando los principios fundamentales incluidos en la Declaración de Helsinki, en sus actualizaciones y en las normativas vigentes (consentimiento informado y derecho a la información, protección de datos personales y garantías de confidencialidad, no discriminación, gratuidad y posibilidad de abandonar el estudio en cualquiera de sus fases). La detección de los casos atípicos, se atendió tanto a la presencia de casos univariantes como multivariantes. La detección de valores atípicos univariantes se realizó mediante la exploración de puntuaciones estandarizadas. Siguiendo los criterios indicados por Hair, Anderson, Tatham y Black (2008), se consideraron valores atípicos a aquellos cuyas puntuaciones estandarizadas presentaran un valor absoluto superior a 4. Para la detección multivariante se computó la distancia de Mahalanobis (Tabachnick \& Fidell, 2007).

\section{Instrumentos}

Escala de Relaciones Intrafamiliares (E.R.I), elaborada por Rivera-Heredia y Andrade (2010) a partir de la Family Environment Scale (FES) de Moos (1981); FACES II (Olson, et al., 1983); Family Assesment Device (FAD) de Epstein y colaboradores (1983); Family of Origin Scale (FOS) de Hovestadt y colaboradores (1985); la escala de Funcionamiento familiar de Bloom (1985); y la Escala de Funcionamiento Familiar (EFF) de Atri y Zetune (1987). La escala es de autorreporte, tipo Likert con rango de 1(totalmente en desacuerdo) a 5 (totalmente de acuerdo) y está integrada por tres dimensiones: unión y apoyo, formada por 11 ítems (p.ej., "los miembros de la familia acostumbran hacer cosas juntos"); expresión, integrada por 22 ítems (p.ej., "en casa acostumbramos expresar nuestras ideas") y dificultades, formada por 23 ítems (p.ej., "hay 
muchos malos sentimientos en la familia"). El coeficiente de fiabilidad $\alpha$ que hemos obtenido en esta escala es de .83 para unión y apoyo, .88 para expresión y .87 para dificultades.

La Escala de Actitudes hacia la Autoridad Institucional en Adolescentes -AAI-A- (Cava, Estévez, Buelga, \& Musitu, 2013). Evalúa las actitudes de los adolescentes hacia las figuras de autoridad e instituciones de autoridad formal, así como las normas sociales. La escala consta de dos dimensiones: actitud positiva hacia la autoridad, formada por 5 ítems, con rangos de respuesta de 1 (nada de cuerdo) a 4 (totalmente de acuerdo), que miden las actitudes de los adolescentes hacia figuras de autoridad formal tales como la policía, (p.ej., "la policía está para hacer una sociedad mejor para todos"); y la actitud positiva hacia la transgresión de normas, compuesta por 4 ítems que evalúan la actitud de los adolescentes hacia las normas escolares y sociales (p.ej., "da igual saltarse las reglas escolares si después no hay castigos"). En esta investigación, los coeficientes de fiabilidad (alfa de Cronbach) fueron de .72 para actitud positiva hacia la autoridad y de .70 para la actitud positiva hacia la transgresión de las normas.

Escala de Conducta Delictiva y Violenta en el Aula (Rubini \& Pombeni, 1992). Evalúa los problemas de comportamiento y victimización en la escuela. La escala está compuesta por dos dimensiones: la conducta violenta/disruptiva, formada por 13 ítems (p.ej., "he pintado o dañado las paredes de la escuela"), y victimización, integrada por 6 ítems (p.ej., “algún(a) compañero(a) me insultó o me pegó"), con rangos de respuesta de 1 (nunca) a 5 (muchas veces). Los coeficientes de fiabilidad $\alpha$ obtenidos en esta escala son de .82 y .73 respectivamente.

Escala de Conducta Violenta en la Escuela (Little, Henrich, Jones, \& Hawley, 2003). Evalúa los tipos de conducta violenta en el contexto escolar y la agresión relacional o indirecta. La escala está compuesta por 25 ítems, con rangos de respuesta de 1 a 4 (nunca, pocas veces, muchas veces y siempre), agrupados en dos dimensiones: la conducta violenta directa o manifiesta, y la agresión relacional o indirecta. La primera está integrada por tres subescalas: agresión manifiesta pura (p.ej., "soy una persona que se pelea con los demás”), agresión manifiesta reactiva (p.ej., "cuando alguien me amenaza, yo lo amenazo también"), y la agresión manifiesta instrumental (p.ej., "amenazo a otros/as para conseguir lo que quiero"). Los coeficientes de fiabilidad $\alpha$ de estas subescalas son de $.77, .78$ y .70, respectivamente. La segunda dimensión está integrada por tres subescalas: agresión relacional pura (p.ej., "soy una persona que cuenta chismes y rumores de los demás"), agresión relacional reactiva (p.ej., "cuando alguien me desagrada lo trato con indiferencia o dejo de hablarle"), y agresión relacional instrumental (p.ej., "para conseguir lo que quiero, chismorreo o cuento rumores sobre los demás"). En nuestro estudio hemos obtenido $\alpha$ de Cronbach de $.77, .71$ y .78 respectivamente.

\section{Análisis de datos}

En primer lugar, se realizó un Análisis Factorial Exploratorio, utilizando el paquete estadístico SPSS (versión 20), y se calculó la fiabilidad de los factores. A continuación, se realizaron análisis confirmatorios utilizando el programa EQS 6.1, con la finalidad de constatar si la estructura factorial obtenida previamente representaba razonablemente los datos. Todos los resultados del ajuste de los modelos se basaron en la solución robusta, debido a la desviación de la normalidad observada en los datos. Esta solución robusta aplica una corrección a la desviación de la multinormalidad de los datos para estimar un $\chi^{2}$ ajustado $(S-B \chi 2)$, errores típicos robustos e índices de ajuste robustos. Finalmente, para analizar la validez convergente de la escala, se analizó la relación entre los factores obtenidos en la escala y las diferentes dimensiones de actitudes hacia la autoridad institucional, la conducta violenta en la escuela, la conducta delictiva y violenta en el aula, y la victimización, mediante análisis de correlación (coeficiente de Pearson) utilizando el paquete estadístico SPSS mencionado anteriormente.

\section{Resultados}

\section{Análisis Factorial Exploratorio y Fiabilidad}

En primer lugar, se obtuvieron los resultados de la prueba de Kaiser-Meyer-Olkin (.87) y de la prueba de Bartlett $\left(\chi^{2}=1180.74, \mathrm{gl}=584, p<.001\right)$ que fueron satisfactorios, por lo que se realizó el Análisis Factorial Exploratorio (AFE). El método utilizado fue el de componentes principales con 
Tabla 1. Análisis factorial exploratorio y confirmatorio

\begin{tabular}{|c|c|c|c|c|}
\hline & \multicolumn{2}{|c|}{ Factorial exploratorio } & \multicolumn{2}{|c|}{ Factorial confirmatorio } \\
\hline & $\mathrm{F} 1$ & F2 & $\mathrm{F} 1$ & F2 \\
\hline 1. En mi familia hablamos con sinceridad & .592 & -.015 & .556 & \\
\hline 2. Nuestra familia no hace las cosas junta & .072 & .546 & & .501 \\
\hline 3. Mis padres me animan a expresar abiertamente mis puntos de vista & .582 & -.023 & .549 & \\
\hline 4. Hay muchos malos sentimientos & -.044 & .608 & & .566 \\
\hline 5. Los miembros de la familia acostumbran hacer cosas juntas & .630 & -.018 & .592 & \\
\hline 6. En casa acostumbramos expresar nuestras ideas & .663 & -.053 & .620 & \\
\hline 7. Me avergüenza mostrar mis emociones frente a la familia & -.044 & .544 & & .514 \\
\hline 8. En nuestra familia es importante para todos expresar nuestras opiniones & .696 & -.041 & .667 & \\
\hline $\begin{array}{l}\text { 9. Frecuentemente tengo que adivinar sobre que piensan los otros miembros de la } \\
\text { familia o sobre cómo se sienten }\end{array}$ & .053 & .542 & & .492 \\
\hline 10. Somos una familia cariñosa & .752 & -.085 & .740 & \\
\hline 11. Mi familia me escucha & .727 & -.119 & .720 & \\
\hline 12. Es difícil llegar a un acuerdo con mi familia & -.073 & .670 & & .639 \\
\hline 13. En mi familia expresamos abiertamente nuestro cariño & .697 & -.083 & .678 & \\
\hline 14. En mi familia, nadie se preocupa por los sentimientos de los demás & -.039 & .685 & & .658 \\
\hline 15. En nuestra familia hay un sentimiento de unión & .663 & -.089 & .652 & \\
\hline 16. En mi familia, yo me siento libre de expresar mis opiniones & .720 & -.146 & .700 & \\
\hline 17. El ambiente en mi familia usualmente es desagradable & -.069 & .662 & & .634 \\
\hline 18. En la familia nos sentimos libres de decir lo que traemos en mente & .635 & -.010 & .600 & \\
\hline $\begin{array}{l}\text { 19. Generalmente nos desquitamos con la misma persona de la familia cuando algo } \\
\text { sale mal }\end{array}$ & -.104 & .570 & & .529 \\
\hline 20. Mi familia es cálida y nos brinda apoyo & .730 & -.109 & .728 & \\
\hline $\begin{array}{l}\text { 21. Cada miembro de la familia apoya con algo en las decisiones familiares } \\
\text { importantes }\end{array}$ & .682 & -.073 & .671 & \\
\hline 22. Encuentro difícil expresar mis opiniones en la familia & -.140 & .660 & & .628 \\
\hline 23. En mi familia a cada quien le es fácil expresar su opinión & .502 & .047 & .469 & \\
\hline 24. Cuando tengo algún problema no se lo platico a mi familia & -.093 & .553 & & .515 \\
\hline 25. Nuestra familia acostumbra hacer actividades en conjunto & .654 & -.039 & .616 & \\
\hline 26. Nosotros somos francos unos con otros & 609 & -.013 & .572 & \\
\hline 27. Es difícil saber cuáles son las reglas que se siguen en nuestra familia & -.002 & .626 & & .585 \\
\hline 28. En mi familia acostumbramos discutir nuestros problemas & .084 & .607 & & .560 \\
\hline $\begin{array}{l}\text { 29. Los miembros de la familia no son muy receptivos para los puntos de vista de } \\
\text { los demás }\end{array}$ & .734 & -.074 & .731 & \\
\hline 30. Los miembros de la familia de verdad nos ayudamos y apoyamos unos a otros & .661 & -.042 & .633 & \\
\hline 31. En mi familia yo puedo expresar cualquier sentimiento que tenga & -.090 & .693 & & .679 \\
\hline 32. Los conflictos de mi familia nunca se resuelven & .737 & -.043 & .716 & \\
\hline 33. En mi familia expresamos abiertamente nuestras emociones & .013 & .587 & & .544 \\
\hline 34. Si las reglas se rompen no sabemos que esperar & .511 & -0.19 & .479 & \\
\hline 35. Las comidas en mi casa, usualmente son amigables y placenteras & .537 & .096 & .492 & \\
\hline 36. En mi familia nos decimos las cosas abiertamente & -.131 & .555 & & .514 \\
\hline
\end{tabular}

Nota. Todos los coeficientes son los significativos (p<.001) F1=Unión, apoyo y expresión, F2=dificultades intrafamiliares

rotación Oblimin. Una vez obtenidos los resultados del análisis factorial, la selección del número de factores se realizó teniendo en cuenta la regla de Kaiser y el gráfico de sedimentación de Cattell. Se observó que había dos factores que mostraban autovalores iniciales superiores a 1 (regla de Kaiser) y que el gráfico de sedimentación mostraba una caída importante de la pendiente a partir del segundo factor. Cabe señalar que del análisis factorial de la escala se suprimió el ítem 28, el cual presentaba saturaciones en las dos dimensiones o factores.

Los dos factores obtenidos explican en conjunto el $41.008 \%$ de la varianza. Analizando el contenido de los ítems que integran cada uno de estos factores, se observa una estructura teóricamente coherente. 


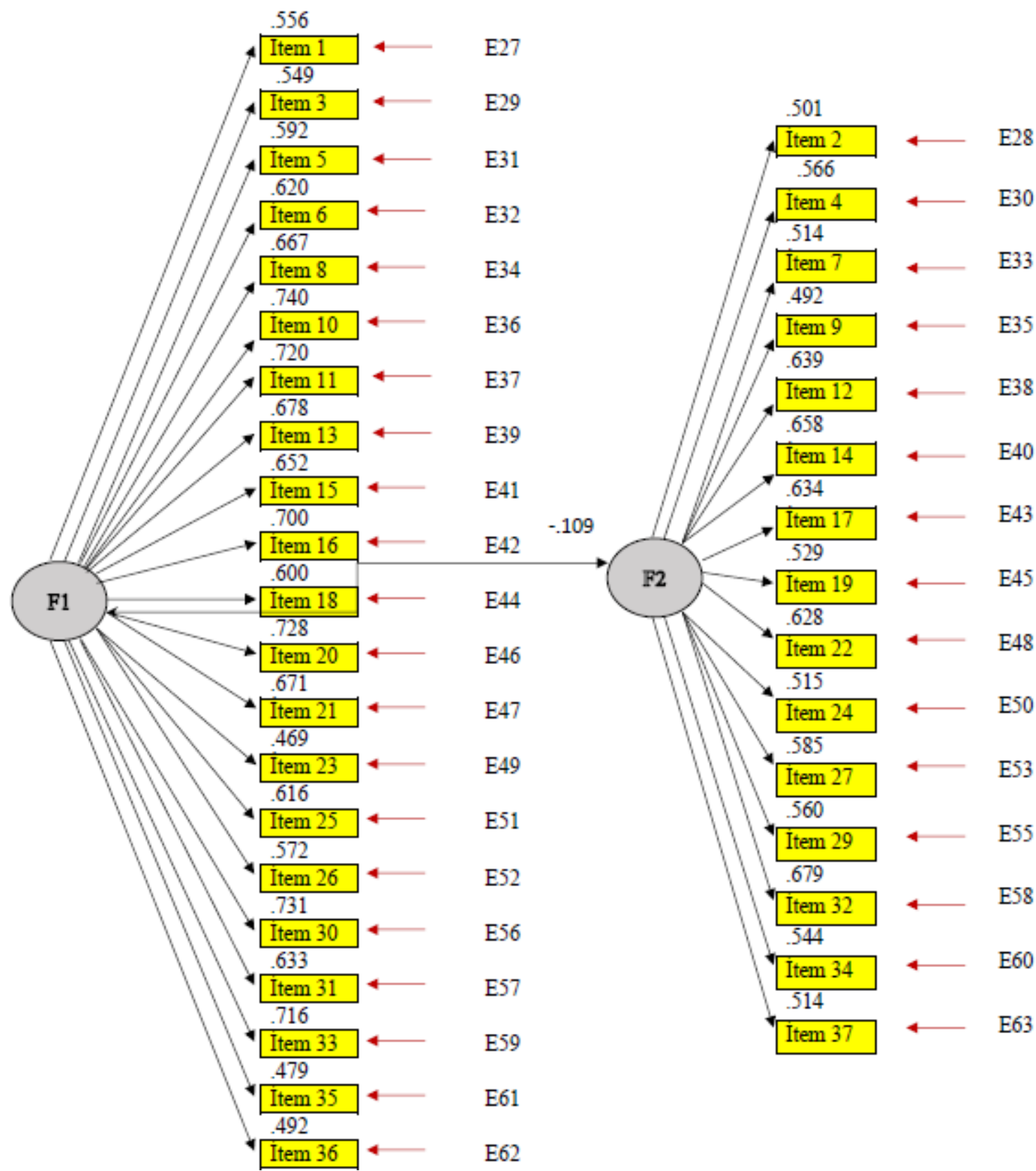

Figura 1. Diagrama confirmatorio de la Escala de Relaciones Intrafamiliar

El primer factor, al que denominamos Unión, Apoyo y Expresión, explica el $25.65 \%$ de la varianza total e incluye a 21 ítems relativos a unión y apoyo familiar, (realizar actividades en conjunto, convivencia y apoyo mutuo) y a la expresión (capacidad de comunicar verbalmente las emociones, ideas y acontecimientos de los miembros de la familia en un ambiente de respeto). El segundo factor, denominado Dificultades, explica el $15.35 \%$ de la varianza y está formado por 15 ítems relacionados con los aspectos no deseados, problemáticos y difíciles dentro de la familia. Todos los ítems muestran saturaciones superiores a .40 . Los coeficientes de consistencia interna (alfa de Cronbach) de los factores obtenidos, muestran niveles altos de fiabilidad., con un alfa de Cronbach de .93 para el factor Unión, apoyo y expresión, de .87 para el factor dificultades, y un alfa de Cronbach de .88 para la escala total. La correlación entre los factores fue de $r=-.077$. A continuación, se presentan los resultados de los factoriales exploratorios y confirmatorios en la Tabla 1.

\section{Análisis Confirmatorio}

Teniendo en cuenta los factores obtenidos con el AFE, se realizó el análisis confirmatorio con el modelo de dos factores correlacionados para los 36 ítems de la escala, con la finalidad de realizar así una validación de la estructura 
Tabla 2. Correlaciones entre relaciones intrafamiliares, actitudes ante la autoridad, conducta delictiva y violencia en el aula y conducta violenta en la escuela.

\begin{tabular}{|c|c|c|c|c|c|c|c|c|c|c|c|c|}
\hline Variable & 1 & 2 & 3 & 4 & 5 & 6 & 7 & 8 & 9 & 10 & 11 & 12 \\
\hline \multicolumn{13}{|l|}{ Relaciones intrafamiliares (E.R.I) } \\
\hline $\begin{array}{l}\text { 1. Relaciones Interfamiliares } \\
\text { (F1) }\end{array}$ & 1 & & & & & & & & & & & \\
\hline $\begin{array}{l}\text { 2. Relaciones Interfamiliares } \\
\text { (F2) }\end{array}$ & $\begin{array}{l}-.077 \\
* *\end{array}$ & 1 & & & & & & & & & & \\
\hline \multicolumn{13}{|l|}{ Actitudes autoridad (AAI-A) } \\
\hline $\begin{array}{l}\text { 3. Actitud positiva hacia la } \\
\text { autoridad institucional }\end{array}$ & $\begin{array}{l}.278 \\
* *\end{array}$ & $\begin{array}{l}-.143 \\
* *\end{array}$ & 1 & & & & & & & & & \\
\hline $\begin{array}{l}\text { 4. Actitud positiva hacia la } \\
\text { transgresión de normas } \\
\text { sociales }\end{array}$ & $\begin{array}{l}-.115 \\
* *\end{array}$ & $\begin{array}{l}.294 \\
* *\end{array}$ & $\begin{array}{c}-.072 \\
* *\end{array}$ & 1 & & & & & & & & \\
\hline Conducta Delictiva Violenta & & & & & & & & & & & & \\
\hline $\begin{array}{l}\text { 5. Conducta } \\
\text { Violenta/Disruptiva }\end{array}$ & $\begin{array}{c}-.223 \\
* *\end{array}$ & $\begin{array}{l}.101 \\
* *\end{array}$ & $\begin{array}{l}-.285 \\
* *\end{array}$ & $\begin{array}{l}.259 \\
* *\end{array}$ & 1 & & & & & & & \\
\hline 6. Victimización & $\begin{array}{c}-.145 \\
* *\end{array}$ & $\begin{array}{l}.081 \\
* *\end{array}$ & $\begin{array}{l}-.173 \\
* *\end{array}$ & $\begin{array}{c}.065 \\
*\end{array}$ & $\begin{array}{l}.448 \\
* *\end{array}$ & 1 & & & & & & \\
\hline \multicolumn{13}{|l|}{ Conducta Violenta Escuela } \\
\hline 7. Agresión manifiesta pura & $\begin{array}{c}-.237 \\
* *\end{array}$ & $\begin{array}{l}.090 \\
* *\end{array}$ & $\begin{array}{c}-.198 \\
* *\end{array}$ & $\begin{array}{l}.244 \\
* *\end{array}$ & $\begin{array}{c}.549 \\
* *\end{array}$ & $\begin{array}{l}.305 \\
* *\end{array}$ & 1 & & & & & \\
\hline $\begin{array}{l}\text { 8. Agresión manifiesta } \\
\text { reactiva }\end{array}$ & $\begin{array}{c}-.194 \\
* *\end{array}$ & .024 & $\begin{array}{c}-.197 \\
* *\end{array}$ & $\begin{array}{l}.268 \\
* *\end{array}$ & $\begin{array}{l}.507 \\
* *\end{array}$ & $\begin{array}{l}.229 \\
* *\end{array}$ & $\begin{array}{c}.613 \\
*\end{array}$ & 1 & & & & \\
\hline $\begin{array}{l}\text { 9. Agresión manifiesta } \\
\text { instrumental }\end{array}$ & $\begin{array}{c}-.170 \\
* *\end{array}$ & $\begin{array}{l}.153 \\
* *\end{array}$ & $\begin{array}{c}-.169 \\
* *\end{array}$ & $\begin{array}{l}.245 \\
* *\end{array}$ & $\begin{array}{l}.429 \\
* *\end{array}$ & $\begin{array}{l}.186 \\
* *\end{array}$ & $\begin{array}{l}.558 \\
*\end{array}$ & $\begin{array}{l}.462 \\
* *\end{array}$ & 1 & & & \\
\hline 10. Agresión relacional pura & $\begin{array}{c}-.163 \\
* *\end{array}$ & $\begin{array}{l}.111 \\
* *\end{array}$ & $\begin{array}{c}-.128 \\
* *\end{array}$ & $\begin{array}{l}.185 \\
* *\end{array}$ & $\begin{array}{l}.326 \\
* *\end{array}$ & $\begin{array}{l}.211 \\
* *\end{array}$ & $\begin{array}{l}.435 \\
* *\end{array}$ & $\begin{array}{l}.366 \\
* *\end{array}$ & $\begin{array}{l}.541 \\
* *\end{array}$ & 1 & & \\
\hline $\begin{array}{l}\text { 11. Agresión relacional } \\
\text { reactiva }\end{array}$ & $\begin{array}{l}-.123 \\
* *\end{array}$ & $\begin{array}{c}-.051 \\
*\end{array}$ & $\begin{array}{l}-.081 \\
* *\end{array}$ & $\begin{array}{l}.105 \\
* *\end{array}$ & $\begin{array}{l}.248 \\
* *\end{array}$ & $\begin{array}{l}.214 \\
* *\end{array}$ & $\begin{array}{l}.357 \\
* *\end{array}$ & $\begin{array}{l}.426 \\
* *\end{array}$ & $\begin{array}{l}.332 \\
* *\end{array}$ & $\begin{array}{l}.452 \\
* *\end{array}$ & 1 & \\
\hline $\begin{array}{l}\text { 12. Agresión relacional } \\
\text { instrumental }\end{array}$ & $\begin{array}{l}-.120 \\
* *\end{array}$ & $\begin{array}{l}.162 \\
* *\end{array}$ & $\begin{array}{c}-.158 \\
* *\end{array}$ & $\begin{array}{l}.236 \\
* *\end{array}$ & $\begin{array}{l}.377 \\
* *\end{array}$ & $\begin{array}{l}.229 \\
* *\end{array}$ & $\begin{array}{l}.473 \\
* *\end{array}$ & $\begin{array}{l}.420 \\
* *\end{array}$ & $\begin{array}{l}.594 \\
* *\end{array}$ & $\begin{array}{l}.619 \\
* *\end{array}$ & $\begin{array}{l}.485 \\
* *\end{array}$ & 1 \\
\hline
\end{tabular}

factorial obtenida (Lloret-Segura, Ferreres-Traver, Hernández- Baeza, \& Tomás-Marco, 2014).

Se utilizó el método de estimación de máxima verosimilitud robusta para todos los análisis, debido a la falta de normalidad multivariada de los datos (coeficiente Mardia =416.07). Teniendo en cuenta las actuales recomendaciones sobre la conveniencia de no utilizar una única medida de ajuste del modelo sino varios índices de diferentes tipos de medidas, se ha considerado, además de la chi-cuadrada (que puede verse afectada por el tamaño de la muestra), los siguientes índices de ajuste: el índice de ajuste comparativo robusto (CFI robusto) y el error de aproximación cuadrático medio (RMSEA). Aunque no deben considerarse como puntos de corte fijos (LloretSegura, et al., 2014), habitualmente son considerados como un buen ajuste del modelo índices del CFI, NFI y NNFI superiores a .95, y aceptables si superan el .90. En el caso del RMSEA se considera un buen ajuste valores inferiores a .05 y un ajuste aceptable valores situados entre .06 y .08 (Lloret-Segura et al., 2014). En este modelo, estos índices mostraron los siguientes valores: $S B x^{2}=1180.74, \mathrm{gl}=584$, $p<.001, C F I=.95, \quad R M S E A=.030$ (.028 - .033). Estos valores, tomados en conjunto, indican un ajuste bueno del modelo y, por tanto, confirman la estructura factorial previamente obtenida con el AFE (Ver Figura 1).

\section{Validez}

Por último, se analizó la validez convergente de la escala considerando las correlaciones entre los dos factores que la componen y las dimensiones de conducta violenta en la escuela, las actitudes hacia la autoridad institucional y conducta delictiva y violenta en el aula (Tabla 2). Se observó que las dos dimensiones de la escala de relaciones intrafamiliares muestran correlaciones significativas con las escalas de actitudes hacia la autoridad institucional, la escala de violencia escolar y la escala de conducta delictiva y violenta en el aula.

La Unión, apoyo y expresión intrafamiliares correlacionan negativa y significativamente con la actitud positiva hacia la transgresión de normas sociales $(r=-.115, \quad p<.01)$, conducta violenta/ disruptiva $(r=-.223, p<.01)$, victimización $(r=-.145$, $p<.01)$, la agresión manifiesta pura $(r=-.237$, 
$p<.01)$, la agresión manifiesta reactiva $(r=-.194$, $p<.01)$, la agresión manifiesta instrumental $(r=-.170$, $p<.01)$, la agresión relacional pura $(r=-.163$, $p<.01)$, la agresión relacional reactiva $(r=-.123$, $p<.01)$ y la agresión relacional instrumental ( $r=-$ $.120, p<.01)$. También, se obtuvo una correlación positiva y significativa con la actitud positiva hacia la autoridad institucional $(r=.278, p<.01)$. Respecto del factor de "dificultades intrafamiliares" se obtuvieron correlaciones positivas significativas con actitud positiva hacia la transgresión de normas sociales $(r=.294$, $p<.01)$, conducta violenta/disruptiva $(r=.101$, $p<.01)$, victimización $(r=.081, p<.01)$, agresión manifiesta pura $(r=.090, \quad p<.01), \quad$ agresión manifiesta instrumental $(r=.153, p<.01)$, agresión relacional pura $(r=.111, p<.01)$ y agresión relacional instrumental $(r=.162, p<.01)$; así como una correlación negativa con actitud positiva hacia la autoridad institucional $(r=-.143, p<.01)$ y con la agresión relacional reactiva $(r=-.051, p<.05)$.

\section{Discusión}

En este estudio se planteó como objetivo presentar evidencias de validez y fiabilidad de la escala de relaciones intrafamiliares (E.R.I.) analizando su estructura factorial exploratoria y confirmatoria. Los resultados obtenidos muestran que la escala tiene unas adecuadas propiedades psicométricas, con una estructura compuesta por dos factores: unión, apoyo y expresión (factor 1) y dificultades familiares (factor 2). Los análisis factoriales realizados muestran una estructura factorial clara y coherente. Se trata, por tanto, de un instrumento fiable y que puede ser de utilidad para los investigadores para analizar las relaciones intrafamiliares. También se ha constatado una gran coherencia teórica en las dos dimensiones y la actitud hacia la autoridad institucional, la conducta disruptiva, victimización y violencia en la escuela. Se constata que los adolescentes con mejores relaciones intrafamiliares positivas expresión, unión y apoyo- muestran también una actitud positiva hacia la autoridad institucional. Esta escala muestra, además, relaciones significativas en la dirección teóricamente esperada, entre las relaciones intrafamiliares y la actitud positiva hacia la trasgresión de normas sociales, la conducta violenta y disruptiva, y la victimización. Estos resultados son muy coherentes con la literatura científica en el sentido de que los alumnos con relaciones intrafamiliares conflictivas es más probable que transgredan las normas, realicen conductas violentas y disruptivas en la escuela y desempeñen un papel dual: agresor/víctima. Estos hallazgos invitan a proponer en futuras investigaciones evaluar las Relaciones Intrafamiliares a partir de la escala que se propone, por sus excelentes propiedades psicométricas.

El factor 1 aporta información sobre las relaciones intrafamiliares en aspectos de comunicación, de calidez emocional, apoyo y unión, aspectos relevantes de la identidad familiar. Las funciones psicológicas de la familia se expresan a través de las relaciones de afecto y del apoyo mutuo entre sus integrantes, esenciales para el bienestar de sus miembros. Estas relaciones mantienen vigente el sentimiento de unidad familiar, es decir la pertenencia a un grupo específico dentro del contexto social más amplio (Cracco \& Costa-Ball, 2019; Estévez \& Musitu, 2016). El cuidado y la pertenencia brindan seguridad y tienen un efecto protector constatado en el bienestar y la salud de los adolescentes (Jiménez et al., 2014; Hunger et al., 2017). La pertenencia, al promover la autoconfianza y la autoestima (García et al., 2018; Musitu \& Callejas, 2017; Rodríguez-Fernández et al., 2016), ayuda al desarrollo de eficacia personal, de una buena adaptación social (Musitu-Ferrer et al., 2019; Bonilla et al., 2017; Carrascosa et al., 2015; Jiménez, Estévez, \& Murgui, 2014) y satisfacción con la vida (Stafford et al., 2016; Zhao et al., 2015). El funcionamiento familiar, en sus diversas mediciones y dimensiones, tiene un papel fundamental en la socialización de los hijos (Smetana, 2017; Stafford et al., 2016). Las dimensiones que se asumen como operativas en todas las culturas son las referidas a la protección, la reciprocidad mutua, la supervisión y el control conductual, el aprendizaje guiado y la participación grupal. Dada la indudable relevancia de la familia para el adecuado ajuste de los adolescentes, confirmado con los hallazgos de investigaciones previamente citadas, se constata el beneficio de la escala, la cual podría utilizarse para promover acciones concretas en el escenario 
de investigación y clínico al proporcionar directrices y orientación que pueden guiar un proceso terapéutico, así como evaluar situaciones de riesgo en grupos o comunidades vulnerables donde el apoyo familiar sea un recurso escaso.

El factor 2 refiere información sobre aspectos negativos, problemáticos o difíciles en el medio familiar. El clima familiar saludable pone de manifiesto la calidad de la comunicación entre padres e hijos, y el grado y la eficiencia de la resolución conflictos entre los miembros de la familia. En contraste, el clima familiar negativo, caracterizado por una comunicación ofensiva o evitativa, falta de claridad en las reglas, inconsistencia parental, prácticas parentales duras con predominio de la crítica, la humillación y la amenaza del afecto, castigos físicos y de otro tipo, $y$ alta frecuencia de conflictos que no se llegan a resolver, tiene una influencia negativa en el ajuste psicosocial de los hijos (Cova et al., 2018; Johnson et al., 2016; Wang \& Kenny, 2014; Wiggins et al., 2015). Se ha aportado evidencia respecto a su relación positiva con, las conductas violentas, delictivas (Garcés-Prettel et al., 2020; Romero-Abrio et al., 2018; Cerezo et al., 2018; Bonilla Castillón et al., 2017; Johnson, et al., 2016; Moratto Vásquez, Berbesí Fernández, Cárdenas Zuluaga, Restrepo Botero, \& Londoño Ardilla, 2015), disruptivas, así como un actitud a la transgresión de normas sociales (Johnson et al., 2016) y a la victimización escolar (Nuñez-Fadda et al., 2020; León-Moreno et al., 2020; Castro, et al., 2019), hallazgos que son coherentes con las correlaciones entre la E.R.I. y estas variables. Al ser una relación bidireccional, estas conductas problemáticas, pueden volverse estresores que la familia tendrá que afrontar, no siempre exitosamente, con los recursos de que disponga (Musitu \& Callejas, 2017).

En cambio, la percepción de un buen clima familiar, caracterizado por la comunicación abierta y fluida, y la percepción del adolescente de la unión y el apoyo familiar, es un factor protector contra las conductas delictivas, las transgresiones de las reglas escolares (Garcés-Prettel et al., 2020; Bonilla, et al., 2017), las de riesgo sexual, antisociales (Johnson et al., 2016), el ciberacoso (Romero-Abrio et al., 2019; Martínez-Ferrer et al., 2019) el uso problemático del móvil (Santana-Vega et al.,2019) y de substancias (Riquelme et al., 2018).
Las relaciones significativas encontradas en esta investigación entre el factor 1 y la actitud positiva hacia la autoridad, confirman que la familia es un agente socializador muy importante que, a través de la calidez emocional, transmite las reglas sociales relevantes para el adolescente (Castro et al., 2019; Bonilla et al., 2017; Estévez \& Jiménez, 2015). La percepción del adolescente de un clima familiar positivo, caracterizado por el afecto y el cuidado o apoyo parental, la intimidad, una baja frecuencia de conflictos, con estrategias de resolución adecuadas, y una comunicación familiar abierta ejercen un papel sustancial en su ajuste psicosocial, situación conectada con una actitud positiva hacia la autoridad institucional (Bonilla et al., 2017; Castro et al., 2019; Cava et al., 2013), el respeto y empatía al medio ambiente (Musitu-Ferrer et al. ,2019). En esa misma línea de análisis, las relaciones familiares positivas desplegarían un efecto protector frente a la violencia escolar, ya que, entre muchos de los méritos de un clima positivo, se potencia la adaptación social de los niños y permite la transmisión de pautas y normas culturales de padres a hijos.

Sin embargo, consideramos que sería importante realizar en un futuro estudios donde, además de seguir avanzando en la comprensión de variables tan relevantes como las actitudes ante la autoridad y la violencia entre iguales, se busque enmarcar la inclusión de otros ámbitos de investigación, como serían los relacionados con la comunidad y aquellos aspectos vinculados con variables individuales.

El presente estudio aporta, en síntesis, un instrumento fiable con adecuadas propiedades psicométricas para la medición de las relaciones intrafamiliares, útil para los investigadores y terapeutas en el contexto iberoamericano. Aporta también las sugerencias de investigaciones en las que se planteaban la importancia de analizar en conjunto las actitudes hacia la autoridad y actitudes hacia las normas, en vinculación con las dimensiones del funcionamiento familiar (Cava et al., 2013).

Sin embargo, es importante reseñar que los resultados expuestos aquí deben interpretarse con cautela, debido a la naturaleza transversal y correlacional de los datos que, como es bien sabido, no permite establecer relaciones causales 
entre las variables. A pesar de estas limitaciones, se mantiene que los resultados obtenidos a partir de la escala E.R.I. que se han expuesto en este trabajo, contribuyen a un mejor conocimiento de las relaciones intrafamiliares y de sus relaciones con otras variables relacionadas con el ajuste del adolescente.

\section{Referencias}

Álvarez, A., Suárez, N., Tuero, E., Núñez, J. C., Valle, A., \& Regueiro, B. (2015). Implicación familiar, autoconcepto del adolescente y rendimiento académico. European Journal of Investigation in Health, Psychology and Education, 5(3), 293-311. https://doi.org/10.30552/ejihpe.v5i3.133

Alves, A. F., Gomes, C. M. A., Martins, A., \& Almeida, L. da S. (2017). Cognitive performance and academic achievement: How do family and school converge? European Journal of Education and Psychology, 10(2), 49-56.

https://doi.org/10.1016/j.ejeps.2017.07.001

Atri, \& Zetune, R. (1993). Confiabilidad y validez del cuestionario de Evaluación del Funcionamiento Familiar EFF. Revista Mexicana de Psicologia, 10(1), 49-55.

Barnes, H. L., \& Olson, D. H. (1985). Parentadolescent communication and the circumplex model. Child Development, 56(2), 438-447. https://psycnet.apa.org/doi/10.2307/1129732

Beavers, R., \& Hampson, B. (2000). The Beavers Systems Model of family functioning. Journal of Family Therapy, 22, 128-143.

Bellón, J. A., Delgado, A., Luna, J., \& Lardelli, P. (1996). Validez y fiabilidad del cuestionario de función familiar Apgar-familiar. Atención Primaria, 18, 153-163.

Bloom, B. L. (1985). A factor analysis of selfreport measures of family functioning. Family Process, 24(2), 235-239. https://doi.org/10.1111/j.15455300.1985.00225.x

Bonilla, C. (2016). Familia, conductas delictivas y violentas en la escuela: Una mirada desde la adolescencia [Tesis doctoral]. Universidad de Guadalajara, Puerto Vallarta, México.

Bonilla, C. E., Nuñez, S. M., Domínguez, R., \&
Callejas, J. E. (2017). Conducta violenta en adolescentes escolarizados: Un modelo estructural predictivo. Universitas Psychologica, 16(4), 1.

https://doi.org/10.11144/Javeriana.upsy164.riam

Calleja, N. (2011) (Comp.). Inventario de escalas psicosociales en México 1984-2005. México: Universidad Nacional Autónoma de México, Facultad de Psicología.

Carrascosa, L., Cava, M. de J., \& Buelga, S. (2015). Actitudes hacia la autoridad y violencia entre adolescentes: Diferencias en función del sexo. Suma Psicológica, 22(2), 102-109. https://doi.org/10.1016/j.sumpsi.2015.08.003

Castro, R., Nuñez, S. M., Musitu, G., \& Callejas, J. E. (2019). Comunicación con los padres, malestar psicológico y actitud hacia la autoridad en adolescentes mexicanos: Su influencia en la victimización escolar. Estudios sobre Educación, 36(1), 113-134. https://doi.org/10.15581/004.36.113-134

Cava, M. J., Estévez, E., Buelga, S., \& Musitu, G. (2013). Propiedades psicométricas de la Escala de Actitudes hacia la Autoridad Institucional en adolescentes (AAI-A). Anales de Psicología, 29(2), 540-548. http://dx.doi.org/10.6018/analesps.29.2.13803 1

Cerezo, F., Ruiz-Esteban, C., Lacasa, C. S., \& Arense, J. J. (2018). Dimensions of parenting styles, social climate, and bullying victims in primary and secondary education. Psicothema, 30(1), 59-65. https://doi.org/10.7334/psicothema2016.360

Comisión Económica Para América Latina y el Caribe (CEPAL). (2016). Panorama Social de América Latina. Recuperado de repositorio.cepal.org/bitstream/handle/11362/ 39965/S1600175_es.pdf

Cova, F., Bustos, C., Rincón, P., Grandón, P., Saldivia, S., \& Inostroza, C. (2018). Propiedades psicométricas de una forma breve del Cuestionario de Parentalidad Alabama en Familias de Preescolares Chilenos. Revista Iberoamericana de Diagnóstico y Evaluación - e Avaliação Psicológica, 51(2), 33-42. https://doi.org10.21865/RIDEP51.2.03

Cracco. C., \& Costa-Ball, C. D. (2019). 
Propiedades psicométricas de la escala de comunicación familiar. Revista Iberoamericana de Diagnóstico y Evaluación - e Avaliação Psicológica, 51(2), 77-86. https://doi.org10.21865/RIDEP51.2.06

Epstein, N., Baldwin, L., \& Bishop, D. (1983). The McMaster Family Assessment Device. Journal of Marital and Family Therapy, 9(2), 171-180. https://doi.org/10.1111/j.17520606.1983.tb01497.x

Estévez, E., \& Jiménez, T. I. (2015). Conducta agresiva y ajuste personal y escolar en una muestra de estudiantes adolescentes españoles. Universitas Psychologica, 14(1), 111-124.

https://doi.org/10.11144/Javeriana.upsy141.caap

Estévez, E., \& Musitu, G. (2016). Intervención psicoeducativa en el ámbito familiar, social y comunitario. Madrid, España: Paraninfo.

Estévez, E., Jiménez, T., \& Cava, M. J. (2016). A cross-cultural study in Spain and Mexico on school aggression in adolescence: Examining the role of individual, family and school variables. Cross-Cultural Research, 50(2), 123-153. https://doi.org/10.1177/1069397115625637

Fernández-Ballesteros, R. y Sierra, B. (1989). Escalas de Clima Social FES, WES, CIES y CES. Madrid, España: TEA.

Freed, R. D., Rubenstein, L. M., Daryanani, I., Olino, T. M., \& Lauren, B. (2017). The relationship between family functioning and adolescent depressive symptoms: The role of emotional clarity. Journal of Youth and Adolescence, 45(3), 505-519. https://doi.org/10.1007/s10964-016-0429-y

Garcés-Prettel, M., Santoya-Montes, Y., \& Jiménez-Osorio, J. (2020). Influence of family and pedagogical communication on school violence. [Influencia de la comunicación familiar y pedagógica en la violencia escolar]. Comunicar, $\quad 63, \quad 77-86$. https://doi.org/10.3916/C63-2020-07

García, O. F., Serra, E., Zacarés, J. J., \& García, F. (2018). Parenting styles and short- and long-term socialization outcomes: A study among Spanish adolescents and older adults. Psychosocial Intervention, 27(3), 153-161. https://doi.org/10.5093/pi2018a21
Grusec, J. E. (2011). Socialization processes in the family: Social and emotional development. Annual Review Psychology, (62), 243-269.

https://doi.org/doi:10.1146/annurev.psych.121 208.131650

Hair, J., Anderson, R., Tatham, R., \& Black, W. (2008). ( $5^{\mathrm{a}}$ ed.). Análisis Multivariante. Madrid, España: Prentice Hall.

Hamilton, E., \& Carr, A. (2016). A systematic review of self-report family assessment measures. Family Process, 55(1), 16-30. http://dx.doi.org/10.1111/famp.12200

Hovestadt, A. J., Anderson, W. T., Piercy, F. P., Cochran, S. W., \& Fine, M. (1985). A family of origin scale. Journal of Marital \& Family Therapy, 11, 287-297.

https://doi.org/10.1111/j.1752-

0606.1985.tb00621.x

Hunger, C., Bornhäuser, A., Link, L., Geigges, J., Voss, A., Weinhold, J., \& Schweitzer, J. (2017). The Experience in Personal Social Systems Questionnaire (EXIS.pers): Development and psychometric properties. Family Process, 56(1), 154-170. https://doi.org/10.1111/famp.12205

Instituto de Política Familiar. (2019). Informe de la evolución de la familia en Iberoamérica. Recuperado de https://fadep.org/wpcontent/uploads/2019/07/F81_Informe-

Evolucion-de-la-Familia-en-Iberoamerica2019.pdf

Jiménez, T. I., Estévez, E., \& Murgui, S. (2014). Ambiente comunitario y actitud hacia la autoridad: Relaciones con la calidad de las relaciones familiares y con la agresión hacia los iguales en adolescentes. Anales de Psicología, 30(3), 1086-1095. https://doi.org/10.6018/analesps.30.3.160041

Jin, Z., Zhang, X., \& Han, Z. R. (2017). Parental emotion socialization and child psychological adjustment among Chinese urban families: Mediation through child emotion regulation and moderation through dyadic collaboration. Frontiers in Psychology, 8(2198), 1-11. https://doi.org/10.3389/fpsyg.2017.02198

Johnson, W. L., Giordano, P. C., Longmore, M. A., \& Manning, W. D. (2016). Parents, identities, and trajectories of antisocial behavior from adolescence to young 
adulthood. Journal of Developmental and Life-Course Criminology, 2(4), 442-465. https://doi.org/10.1007/s40865-016-0044-3

Little, T. D., Henrich, C. C., Jones, S. M., \& Hawley, P. H. (2003). Disentangling the "whys" from the "whats" of aggressive behaviour. International Journal of Behavioral Development, 27(2), 122-133. https://psycnet.apa.org/doi/10.1080/01650250 244000128

León-Moreno, C., Callejas-Jerónimo, J. E., Suarez-Relinque, C., Musitu-Ferrer, D., \& Musitu-Ochoa, G. (2020). Parental socialization, social anxiety, and school victimization: A mediation model. Sustainability, 12(7), 1-12. doi:10.3390/su12072681

Lloret-Segura, S., Ferreres-Traver, A., HernándezBaeza, A., \& Tomás-Marco, I. (2014). El análisis factorial exploratorio de los ítems: Una guía práctica, revisada y actualizada. Anales de Psicología, 30(3), 1151-1169. http://dx.doi.org/10.6018/analesps.30.3.19936 1

Martínez-Ferrer, B., León-Moreno, C., MusituFerrer, D., Romero-Abrio, A., CallejasJerónimo, J., \& Musitu-Ochoa, G. (2019). Parental socialization, school adjustment and cyber-aggression among adolescents. International Journal of Environmental Research and Public Health, 16(4005), 1-14. doi:10.3390/ijerph16204005

Martínez-Pampliega, A., Merino, L., Iriarte, L., \& Olson, D. (2017). Psychometric properties of the spanish version of the Family Adaptability and Cohesion Evaluation Scale IV. Psicothema, 29(3), 414-420.

doi: $10.7334 /$ psicothema2016.21

Moos, R. H., \& Moos, B. S. (1981). Family Environment Scale Manual. Palo Alto, CA: Consulting Psychologist Press.

Moratto Vásquez, N. S., Berbesí Fernández, D. Y., Cárdenas Zuluaga, N., Restrepo Botero, J. C., \& Londoño Ardila, L. F. (2015). La familia como factor predictor de la intimidación escolar en Antioquia (Colombia). Revista Clínica de Medicina de Familia, 8(2), 97-102. http://dx.doi.org/10.4321/S1699695X2015000200002
Musitu, G., \& Callejas, J. E. (2017). El modelo de estrés familiar en la adolescencia: Mefad. International Journal of Developmental and Educational Psychology. Revista INFAD de Psicología, 1(1), 11-19. https://doi.org/10.17060/ijodaep.2017.n1.v1.8 94

Musitu-Ferrer, D., León-Moreno, C., CallejasJerónimo, J. E., Esteban-Ibáñez, M., \& Musitu-Ochoa, G. (2019). Relaciones entre estilos de socialización parental, empatía y conexión con la naturaleza: Sus implicaciones en el ambienormntalismo. International Journal of Environmental Research and Public Health, 16(2461), 1-20. doi:10.3390/ijerph16142461

Nuñez-Fadda, S. M., Castro-Castañeda, R., Vargas-Jiménez, E., Musitu-Ochoa, G., \& Callejas-Jerónimo, J. E. (2020). Bullying victimization among Mexican adolescents: Psychosocial differences from an ecological approach. International Journal of Environmental Research and Public Health, 17(4831), 1-16. doi:10.3390/ijerph17134831

Olson, D. H., McCubbin, H. I., Barnes, H. L., Muxen, M. J., Larsen, A. S., \& Wilson, M. A. (1983). Families, what makes them work. Newsbury, California: Sage Publications.

Oppenheimer, C. W., Ladouceur, C. D., Waller, J. M., Ryan, N. D., Allen, K. B., Sheeber, L., Forbes, E. E., Dahl, R. E., \& Silk, J. S. (2016). Emotion socialization in anxious youth: Parenting buffers emotional reactivity to peer negative events. Journal of Abnormal Child Psychology, 44(7), 1267-1278. doi:10.1007/s10802-015-0125-5

Riquelme, M., Garcia, O. F., \& Serra, E. (2018). Desajuste psicosocial en la adolescencia: socialización parental, autoestima y uso de sustancias. Anales de Psicología, 34(3), 536544.

https://doi.org/10.6018/analesps.34.3.315201

Rivera-Heredia, M. E., \& Andrade, P. (2010) Escala de evaluación de las Relaciones Intrafamiliares (E.R.I.). Uaricha Revista de Psicología 9, 19, 1-19.

Rodríguez-Fernández, A., Ramos-Díaz, E., Ros, I., Fernández-Zabala, A., \& Revuelta, L. (2016). Bienestar subjetivo en la adolescencia: El papel de la resiliencia, el autoconcepto y el 
apoyo social percibido. Suma Psicológica, 23(1), 60-69.

https://doi.org/10.1016/j.sumpsi.2016.02.002

Romero-Abrio, A., Martínez-Ferrer, B., Musitu-

Ferrer, D., León-Moreno, C., VillarrealGonzález, M. E., \& Callejas-Jerónimo, J. E. (2019). Family communication problems, psychosocial adjustment and cyberbullying. International Journal of Environmental Research and Public Health, 16(2417), 1-13. doi:10.3390/ijerph16132417

Romero-Abrio, A., Musitu-Ochoa, G., CallejasJerónimo, J. E., Sánchez-Sosa, J. C., \& Villarreal-González, M. E. (2018). Factores predictores de la violencia relacional en la adolescencia. Liberabit, 24(1), 29-43. https://dx.doi.org/https://doi.org/10.24265/libe rabit.2018.v24n1.03

Rubini, M., \& Pombeni, M. L. (1992). Cuestionario de conductas violenta en el aula. Mimeo. Universidad de Bolonia, Facultad de Ciencias de la Educación. Área de Psicología Social.

Santana-Vega, L., Gómez-Muñoz, A., \& Feliciano-García, L. (2019). Uso problemático del móvil, fobia a sentirse excluido y comunicación familiar de los adolescentes. Comunicar, 59(27), 39-47.

https://doi.org/10.3916/C59-2019-04

Santos, J., Muñoz, Á., Juez, P., \& Cortiñas, P. (2003). Diseño de encuestas de estudio de mercado. Técnicas de muestreo y análisis multivariante. Madrid, España: Editorial Ramón Areces.

Schmidt, V., Barreyro, J. P., \& Maglio, A. L. (2010). Escala de evaluación del funcionamiento familiar FACES III: ¿Modelo de dos o tres factores? Escritos de Psicología, 3(2), 30-36.

Simpson, E. G., Vannucci, A., \& Ohannessian, C. M. (2018). Family functioning and adolescent internalizing symptoms: A latent profile analysis. Journal of Adolescence, 64, 136145.

https://doi.org/doi:10.1016/j.adolescence.2018 .02 .004

Smetana, J. G. (2017). Current research on parenting styles, dimensions, and beliefs. Current Opinion in Psychology, 15, 19-25. https://doi.org/10.1016/j.copsyc.2017.02.012
Smilkstein, G., Ashworth, C., \& Montano, D. (1982). Validity and reliability of the Family APGAR as a test of family function. Journal of Family Practice, 15, 303-11.

Stafford, M., Kuh, D. L., Gale, C. R, Mishra, G., \& Richards, M. (2016). Parent-child relationships and offspring's positive mental wellbeing from adolescence to early older age. Journal of Positive Psychology, 11(3), 326-337. doi:10.1080/17439760.2015.1081971

Tabachnick, B. G., \& Fidell, L. S. (2007). Using multivariate statistics, $6^{\text {th }}$ ed. CA, USA: Pearson.

Thomassin, K., Suveg, C., Davis, M., Lavner, J. A., \& Beach, S. R. H. (2017). Coparental affect, children's emotion dysregulation, and parent and child depressive symptoms. Family Process, 56(1), 126-140. https://doi.org/10.1111/famp.12184

Ullmann, H., Maldonado Valera, C., \& Rico, C. N. (2014). La evolución de las estructuras familiares en América Latina, 1990-2010. Los retos de la pobreza, la vulnerabilidad y el cuidado. Santiago de Chile: Naciones Unidas, CEPAL, UNICEF. Recuperado de https://www.unicef.org/lac/S2014182_es.pdf

Wang, M.- Te, \& Kenny, S. (2014). Longitudinal links between fathers' and mothers' harsh verbal discipline and adolescents' conduct problems and depressive symptoms. Child Development, 85(3), 908-923. https://doi.org/10.1111/cdev.12143

Whittle, S., Vijayakumar, N., Dennison, M., Schwartz, O., Simmons, J. G., Sheeber, L., \& Allen, N. B. (2016). Observed measures of negative parenting predict brain development during adolescence. PloS ONE, 11(1), e0147774.

http://dx.doi.org/10.1371/journal.pone.0147774

Wiggins, J. L., Mitchell, C., Hyde, L. W., \& Monk, C. S. (2015). Identifying early pathways of risk and resilience: The codevelopment of internalizing and externalizing symptoms and the role of harsh parenting. Development and Psychopathology, 27(4pt1), 1295-1312. https://doi.org/10.1017/S0954579414001412

World Medical Association. (2013). World Medical Association Declaration of Helsinki. 
Ethical principles for medical research involving human subjects. Journal of the American Medical Association, 310, 21912194.

Zhao H., Xu Y., Wang F., Jiang J., \& Zhang X. (2015). Influence of parent-adolescent conflict frequency on adolescent family satisfaction and self-satisfaction in China: Conflict coping tactics as moderators. Psychological Report, 117(3), 897-915.

https://doi.org/10.2466/21.10.PR0.117c28z7 . 